\title{
ABUNDANCES OF METAL-POOR STARS AND THE FORMATION OF THE HALO
}

\author{
R. CAYREL \\ Observatoire de Paris et C.N.R.S. (URA 335) \\ 61 , avenue de l'Observatoire \\ 75014 Paris, France
}

\begin{abstract}
The origin of the elements in the early Galaxy has been thought to be fairly well understood as primordial matter polluted by ejectae of type II supernovae. Some recent observational results have led to reconsider in more detail this statement. As a result, it is confirmed in some respects, but curiously, when the same metallicity can be found both in the halo and in the thick disk, the stars contaminated by SN Ia ejectae are more the halo stars than the thick disk stars.
\end{abstract}

\section{Introduction}

The greatest breakthrough in the field covered by this joint discussion, which has occurred in the last years, is the detailed analysis of a bunch of halo stars in the metallicity range $-4<[\mathrm{Fe} / \mathrm{H}]<-2.5$ (McWilliam et al. 1995, Sneden et al. 1996, Ryan et al. 1996), made possible by the discovery of over 50 extremely metal-poor stars by the H\&K Ca II lines survey of Beers, Preston and Shectman (BPS survey in brief), (Beers et al., 1992).

Because the subject has been covered by the principal author of this breakthrough in the first talk, this morning, I shall speak of other things in my talk.

I briefly, mention in section 2 , a new controversy on the nature of the process which has produced the bulk of the neutron-capture elements in the extreme population II. In section 3, I discuss a fairly new discovery, concerning the $\alpha$-elements over iron peak elements ratio in metal-poor stars. Section 4 deals with the question of the how to properly formulate the problem of the chemical evolution of the Galaxy. Section 4 concludes the talk.

\section{2. r-process versus $s$-process: reopening the case?}

It has been known from many years (Truran, 1981), that there was strong indications that neutroncapture elements in very metal-poor stars were chiefly produced by the $r$-process only, whereas it is well established that both processes $r$ and $s$ have contributed to the solar system chemical composition. The case has been reopened by a paper by Magain (Magain, 1995), who claimed that the very metal-poor star HD 140283 had most of its barium generated by the $s$-process. The argument is based on the analysis of the profile of the $4554 \AA$ line. The width of the line depends upon the proportion of $r$-process and $s$-process barium, the hyperfine structure of the odd isotopes producing an extra-broadening of the line. Contrary to expectation, the width of the line was favoring the $s$-process rather than the $r$-process. Another piece of evidence, in the opposite direction comes from the analysis of the even more metal-poor star CS 22892-052(Sneden et al., 1996), which is very enhanced in neutron-capture elements with respect to iron, and which wonderfully fits the proportions of solar $r$-elements only. It was worth studying more carefully if the conclusions of Magain, at the limit of the $\mathrm{S} / \mathrm{N}$ ratio of the data, were inescapable. This was done by P. François and W. Gacquer, with new observations taken at CFHT. The results will be presented next week at Symposium 187, where you are invited to hear about the issue.

62 
3. The new $[\alpha / \mathrm{Fe}]$ mess

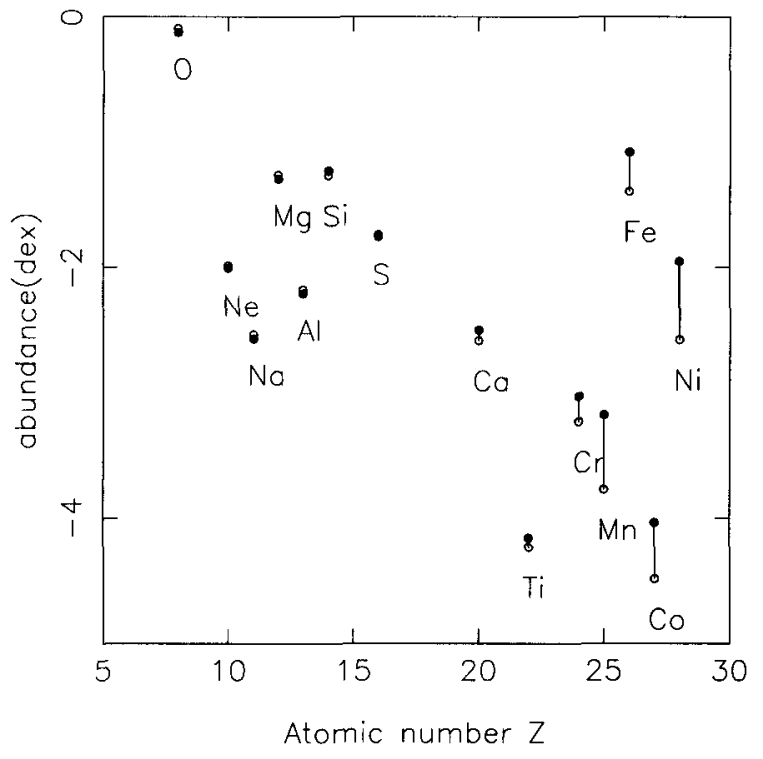

Figure 1. According to Tsujimoto et al. (1995) the proportion of several elements by mass, with respect to their sum, is shown (i) for the ejectae of SNe II, averaged over an initial mass function of Salpeter, between 10 and $50 M_{\odot}$ (open circles); (ii) for the same ejectae mixed with the ejectae of SNe Ia in the proportion of 0.15 SN Ia per SN II. This is about the ratio producing the best fit to the solar composition. Note that the elements up to $\mathrm{Ca}$ are little affected by the contribution or no-contribution of SNe Ia, whereas the iron-peak elements are strongly affected.

One of the pillar of stellar nucleosynthesis is the fact that the $\alpha$-elements are less deficient than iron by about $0.4 \mathrm{dex}$ in metal-poor stars, with respect to the solar composition. This fact has recently been shown to be true also for extremely metal-deficient stars (McWilliam et al., 1995). The current interpretation from 1980 (Tinsley, 1980), is that pop. I has been enriched both by the yields of SNe II and SNe Ia, whereas pop. II was enriched by SNe II only, because of the time-lag for the production of SNe Ia. The comparison of the yields predicted for the two types of supernovae, is the base for this interpretation, which can be even made more refined, according to the $\alpha$-element under consideration, as they have each a different production ratio. Figure 1 shows this effect. The yields are from Tsujimoto et al. 1995. The circles represent the fractional abundances for pure SNe II ejectae, averaged over a Salpeter initial mass function. The fractional abundance is by mass of the element with respect to all the elements given, in decimal exponent.The dots are the same data for a mixture of SNe II and SNe Ia, with 0.15 SN Ia per SN II (approximately what reproduces the solar composition). It is clear that the proportions are not affected from $\mathrm{O}$ to $\mathrm{Ti}$, whereas the iron peak elements are enhanced by the SN Ia contribution. This behaviour is statistically verified, but not in all details. For example, $[\mathrm{Cr} / \mathrm{Fe}]$ and $[\mathrm{Ni} / \mathrm{Fe}]$ should be different in metal-poor stars ( with the usual notation $[\mathrm{X}]=\log (X($ star $) / X($ Sun $)))$ and this is not the case. Recently attention has been drawn onto apparent exceptions to this rule. (King, 1997) found that the common proper motion pair $\mathrm{HD} 134439$ and 134440 , which have $[\mathrm{Fe} / \mathrm{H}]=-1.5$ and extreme kinematical properties $(\mathrm{U}=310, \mathrm{~V}=-467, \mathrm{~W}=44$, in $\mathrm{km} / \mathrm{s}$ with respect to the $\mathrm{LSR})$, have practically $[\alpha-\mathrm{el} / \mathrm{Fe}]=0$. They have interpreted the fact as evidence for an accreted star, with a different chemical history. Carney et al. 1997 have discovered another $\alpha$-element singular halo star $\mathrm{BD}+80^{\circ} 245$, which is even $\alpha$ element-poor instead of being $\alpha$-element-rich. Its kinematics is ( $U=-173, V=-333, W=+255$ ), also fairly extreme, and has $[\mathrm{Fe} / \mathrm{H}]=-1.8$. The authors also suggest that the star had a chemical history different from ordinary galactic halo stars. The star is also overdeficient in barium. The story could end there if Nissen\&Schuster (1997) had not found, in studying halo and thick disk stars of overlapping metallicity, that $[\alpha-\mathrm{el} / \mathrm{Fe}]$ was just the contrary of what one may have naivvely 

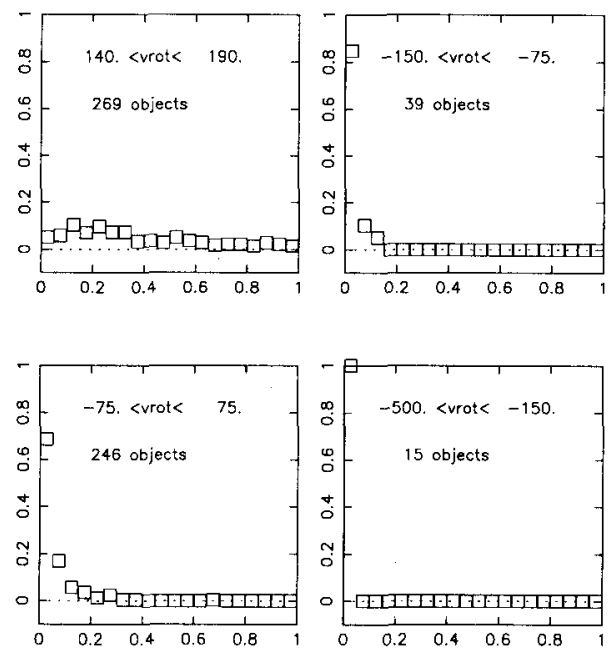

Figure 2. Metallicity histogram as a function of angular momentum. The sample is that of Carney et al. 1994. The abcissa is the metallicity, in fraction of the solar metallicity. The ordinate is the fraction of objets in each bin. The size of the bins is $0.05 Z_{\odot}$. Note the striking difference between the histogram of stars with a thick disk kinematics $\left(140 .<V_{\text {rot }}<190\right.$.) and the 3 histograms of the halo stars. Note also the continuous increase of the first bin fraction when the angular momentum decreases.

expected. In kinematically selected halo stars two thirds of the objects have $[\alpha-\mathrm{el} / \mathrm{Fe}] \approx 0$, whereas it has the high value 0.3 to 0.4 (usually found in the halo) in the thick-disk stars! We are of course here also at metallicities unusally large for halo stars. This time, it becomes difficult to slip the problem under the carpet in invoking an "accretion" event. The stars have not a singular kinematics, and as suggested by the authors, the meaning of this observation might be that halo regions, with a particularily low rate of astration, may well have formed stars with a substantial contamination by $\mathrm{SNe}$ Ia, even at low metallicy. Actually the fit of the abundances is very consistent with the $\mathrm{SNe}$ Ia yields for $\mathrm{Mg}, \mathrm{Si}$ and $\mathrm{Ca}$. A few globular clusters, as Palomar 12 and Ruprecht 106 share this property, and are reputed 2 or $3 \mathrm{Gyr}$ younger than the bulk of globular clusters. Therefore, the lesson is that low-metallicity does not necessarily means an age large enough to have avoided contamination by SN Ia, and that a period has existed in which the halo was still forming stars, after the disk had started star formation. The author of this presentation does not believe that the prograde or retrograde motion of the stars is a key element in this $\alpha$ element issue.

We present in fig.2, the evolution of the metallicity histogram of the stars of the (Carney et al., 1994) sample, as a function of the angular momentum of the objects. The sample with velocity rotations typical of the thick disk shows a fairly flat histogram, with a mean metallicity half solar, but a shallow maximum at one fifth solar. The typical halo, taken by the selection $-75<V_{\text {rot }}<+75$, in order to get rid of thick disk contamination, has a completely different histogram, with a sharp peak in the first three bins, and practically no star beyond metallicity 0.2 solar. The bulk of the retrograde halo has a similar distribution, more sharply peaked ( 85 per cent in the first bin). Finally the extreme retrograde halo, $V_{\text {rot }}<-150$, has all the objects in the first bin (note however that the total number of objects becomes small and prone to statistical fluctuation). The conclusion is that the retrograde halo is, on average, very metal-poor, and is the most primitive component of our Galaxy, even if it has produced later, a few rare $\alpha$-el. rich objects.

\section{A global view of galaxy evolution}

The concept of accreted system is more and more heard in the context of galactic evolution. Actually there is not a single proved example of accretion, in a strict acception of this term, which is the encounter with a body with a positive energy, ending up by dynamical friction, as gravitationaly bound to the Galaxy. The example of the Sagittarius spheroidal galaxy (Ibata et al., 1994), often 
uncorrectly described as an accretion event, is not an accretion, but the merging of a substructure which has always been there. The mere fact that the kinematics of halo stars shows that the gas from which they have originated was moving both in prograde and retrograde motions, proves that this gas had to be clumped in smaller substructures, as expected from cosmology. Therefore, the question is not if a given object has been accreted or not but how long it has been trapped in a substructure before dissolving into the general galactic field. It is of course beyond the scope of this talk to further elaborate on this subject, but the point deserved to be mentioned.

\section{Conclusion}

- The great event of these last years is the access to a stellar population substantially more metalpoor than the most metal-poor globular clusters, in the range $-4<[\mathrm{Fe} / \mathrm{H}]<-2.5$.

- The case for a formation of the neutron-capture elements only by the $r$-process in very metal-poor stars has been strengthened by the discovery of a very metal-poor star with a superb enhanced spectrum of $r$-process rare earths (CS 22892-052). On the other side, the analysis of the profile of a barium line in HD 140283 has opened a controversy on which you will hear during the symposium 187 (W. Gacquer), next week.

- The statement that $[\alpha-\mathrm{el} / \mathrm{Fe}]$ is a function of $[\mathrm{Fe} / \mathrm{H}]$ in the Galaxy, has been recently shaken by counter-examples. It must be admitted that a high $[\alpha-\mathrm{el} / \mathrm{Fe}]$ is the signature of an early bird in the Galaxy, whatever is its metallicity, which, at any time, has a strong scatter in the Galaxy.

- The concept of "accretion" must be used more cautiously. It is improperly used for example for the Sagittarius Ibata\&Gilmore spheroidal galaxy: because the dynamical study of the Milky Way Sagitarius system shows that it is a bound system, the term of "merging" of a substructure would be more appropriate. For the chemical history of the Galaxy the important question is then to know how long the substructures have evolved as independant systems, before dissolving into the general field.

\section{References}

Beers T.C., Preston G.W., Shectman S.A, 1992, AJ, 103,1987

Carney, B.W., Latham, D.W., Laird, J.B., Aguilar, L.A., 1994, AJ, 107, 2240

Carney, B.W., Wright, J.S., Sneden, C., Laird, J.B., Aguilar, L.A., Latham, D.W., 1997, AJ, 114, 363

Ibata, R.A., Gilmore, G., Irwin, M.J., 1994 ,Nature, 370, 194

King, J.R., 1997, $A J, 113,2302$

McWilliam A., Preston G.W., Sneden C., Shectman S.A., 1995, $A J, 109,2757$

Magain, P.,1995, $A \& A, 297,686$

Ryan S.G., Norris J.E., Beers T.C., 1996, ApJ, 471, 254

Sneden C., McWilliam A., Preston G.W., Cowan J.J., Burris D.L., Armosky B.J., 1996, ApJ, 467, 819

Tinsley, B., 1980, Fundam. Cosmic Phys., 5, 287

Truran, J.W., 1981, $A \& A, 97,391$

Tsujimoto, 'T., Nomoto, K., Yoshii, Y., Hashimoto, M., Yanagida, S., Thielemann, F.-K., 1995, MNRAS, 277, 945 